\title{
CircC16orf62 promotes hepatocellular carcinoma progression through the miR-138-5p/PTK2/AKT axis
}

Shuai Zhang ${ }^{1,2,3}$, Yuan Lu ${ }^{4}$, Hong-Yu Jiang ${ }^{3}$, Zhi-Mei Cheng ${ }^{2}$, Zi-Jing Wei', Yun-Hao Wei', Ting Liư ${ }^{4}$, Bai-Juan Xia ${ }^{5}$, Xu-Ya Zhao ${ }^{1}$, Yu Huang ${ }^{1}$, Xun Zou', Rong Liu' ${ }^{7}$ and Shi Zhou ${ }^{2}{ }^{2}$

\begin{abstract}
Circular RNA (circRNAs) functions vital in the pathogenesis and progression of hepatocellular carcinoma (HCC). However, the expressions and functions of certain circRNAs on metastasis and proliferation of that cancer is still unclear. Bioinformation analysis and qRT-PCR indicated that CircC16orf62 was prominent upregulated in HCC of which the expression level was positively associated to cancer's malignant progression. Gain or loss-of-function studies indicated that the reduction of CircC16orf62 expression promotes the proliferation, invasion, and glycolysis of HCC in vitro and in vivo. The bioinformatic analysis found that miR-138-5p and PTK2 were the downstream target of CircC16or62. Then, the FISH(Fluorescence immunoin situ hybridization) and cell nucleoplasmic separation determined that CircC16orf62 located in the cell cytoplasm. Plasmid vectors or siRNAs were used to change the expression of CircC16orf62, miR-138-5p, and PTK2 in PC cell lines. CircC16orf62 functioned as a molecular sponge for miR-138-5p, and a competitive endogenous RNA for PTK2, promoting AKT/mTOR pathway activation. Our observations lead us to conclude that CircC16orf62 functions as an oncogene in HCC progression, behaving as a competitive endogenous RNA for miR-138-5p binding, thus activating the AKT/mTOR pathway. In conclusion, CircC16orf62 is an oncogene through the miR-138-5p/PTK2/Akt axis in HCC cells, indicating CircC16orf62 can be a therapeutic target with potentiality for liver cancer and a predictive marker for people with HCC.
\end{abstract}

\section{Introduction}

Ninety percent of patients with primary liver carcinomas suffer hepatocellular carcinoma (HCC) ${ }^{1}$ which possesses high morbidity and mortality rates, causing malignancy. Although vital achievements are made in therapies for HCC, the 5-year livability stays low. What needs to mention is that, the molecular pathogenesis and therapeutic targets of the cancer is still unclear. So it is obvious that we need to understand its pathogenic process and regulatory

\footnotetext{
Correspondence: Shi Zhou (shizhou0128@126.com)

${ }^{1}$ Department of Interventional Radiology, The Affiliated Cancer Hospital of Guizhou Medical University, Guiyang 550000, P.R. China

${ }^{2}$ Department of Interventional Radiology, the Affiliated Hospital of Guizhou Medical University, Guiyang 550004, P.R. China

Full list of author information is available at the end of the article

These authors contributed equally: Shuai Zhang, Yuan Lu
}

Edited by B. Rotblat mechanisms. RNAs regulate cell activities including circular RNAs, microRNAs, and other noncoding RNAs ${ }^{2}$.In the 1970s, circular RNA was first discovered and confirmed in a virus ${ }^{3}$. For the limits of detection, we viewed circRNA as a situation of incorrect splicing while in exon transcription $^{4}$. So the existence had not got enough attention ${ }^{5}$. Because of its special molecular biological structure, circular RNA has been ignored by biologists, although it may play an extremely critical role ${ }^{6}$. Expression of CircRNAs can be found widely in human cells with various expression levels in different tissues ${ }^{7}$. Researches suggest that malignancy is regulated by circRNAs, such as the proliferation of cancer cells and etc ${ }^{8}$. Those researches also investigated the function of circRNAs in cancers ${ }^{9}$. For instance, the proliferation and migration of cervical cancer cells is regulated by circCLK 3 which modulates the FoxM1 gene expression

\section{(c) The Author(s) 2021}

(c) (i) Open Access This article is licensed under a Creative Commons Attribution 4.0 International License, which permits use, sharing, adaptation, distribution and reproduction cc) in any medium or format, as long as you give appropriate credit to the original author(s) and the source, provide a link to the Creative Commons license, and indicate if changes were made. The images or other third party material in this article are included in the article's Creative Commons license, unless indicated otherwise in a credit line to the material. If material is not included in the article's Creative Commons license and your intended use is not permitted by statutory regulation or exceeds the permitted use, you will need to obtain permission directly from the copyright holder. To view a copy of this license, visit http://creativecommons.org/licenses/by/4.0/. 
by sponging miR-320 $\mathrm{a}^{10}$. The proliferation of gastric cancer and esophageal carcinoma cells were promoted by the CircAmotl1 via the host gene Amotl1 which can be considered as an oncogenic factor ${ }^{11}$. CircRNAs sponge the target miRNAs like competing endogenous RNAs (ceRNAs). Recently, it is been reported that the proliferation and invasion of esophageal squamous cell carcinoma can be improved by FUT8 which helps sponge miR-570-3p to promote Krupple-like Factor $10^{12}$. The circRNA AKT1 can sponge miR-942-5p to affect the AKT1 expression, by enhancing the cervical cancer progression ${ }^{13}$. Also, the RNA type we study here interacts with transcription factors and proteins to build and regulate circRNPs. Circular RNA circCUL3 was revealed that combined with STAT3 to accelerate the Warburg effect progression of gastric cancer through regulating the HK2 expression ${ }^{14}$. The current study indicates that circRNAs function vital for the initiation, migration, and invasion of $\mathrm{HCC}^{15,16}$, but the mechanism on regulating malignancy remains unclear ${ }^{17}$.

Here we examined the differentially expressed circRNAs which noticeably downregulated in HCC tissues and figured out circC16orf62 over-expression significantly attenuated its malignancy. And we investigated the possible targeted miRNAs and noticed that circC16orf62 sponges oncogenic miR-138-5p like a ceRNA, enhancing oncogene PTK2 expression and attenuating the malignancy.

\section{Materials and methods HCC specimens}

The experiment here got approval from the Ethics Committee of Guizhou Medical University. Eighty-eight pairs of surgical or biopsied HCC and adjacent non-tumor specimens in 2015 from 2020 were obtained and they were gained with the informed consent of patients. Diagnoses of patients are according to the practice guidelines of the American Association for the Study of Liver Diseases (AASLD). Tissue RNA was gained by RNeasy Mini Kit (Qiagen, Hilden, Germany), and measurement on RNA concentrations was by NanoDrop 2000 spectrophotometer (ThermoScientific, Waltham, MA). The contained circRNAs were enriched and digested with RNase A and reversely transcribed into cRNA by fluorescent reagents and random primers. This study was approved by the Ethics Committee of The Affiliated Cancer Hospital of Guizhou Medical University.

\section{Cell culture}

Human HCC BEL7402, QGY7701, SMMC7721, Huh-7, Hep3B, HepG2, non-tumor human liver cells were got from American Type Culture Collection (ATCC, Manassas, VA, USA), culturing in DMEM and RPMI-1640 containing $20 \%$ fetal bovine serum (FBS), 100 units $/ \mathrm{ml}$ of penicillin, and $100 \mu \mathrm{g} / \mathrm{ml}$ of streptomycin at $37^{\circ} \mathrm{C}$ in an incubator of $5 \% \mathrm{CO}_{2}$.

\section{siRNA and plasmid transfection}

Shanghai Hanheng Biotech (Shanghai, China) provided the plasmid of CircC16orf62-shRNA, miR-138-5pmimic, and the two's negative control miRNAs. The siRNA, miRNA mimic, and miRNA inhibitor were transfected into the HCC cell used by Lipofectamine 3000 (ThermoFisher). The leti-virus plasmid was transfected into the HCC cells with polybren. And the transfection lentivirus content was according to the individual multiplicity of infection (MOI) in the presence of $5 \mu \mathrm{g} / \mathrm{ml}$ puromycin.

\section{Polymerase chain reaction}

We collected different cells and RNAs extraction by Trizol reagent (Invitrogen). Superscrpt II kit (Invitrogen) reversely transcribes the RNA we got into cDNA. The evaluation on relative expression levels of target gene RNA transcripts were got by qRT-PCR of SYBR Green mix (Takara, Dalian, China).

\section{Western blot}

Protein samples isolated from indicated pancreatic cancer cells were loaded onto SDS-PAGE and transferred to PVDF membranes. Membranes were blocked with 5\% skimmed milk, incubated with primary antibodies HK2 (Anti-HK2, 1:1000, ab209847, Abcam), PKM2 (AntiPKM2, 1:1000, ab85555, Abcam), GUT1(Anti-GUT1, 1:1000, ab115730, Abcam), PTK2 (Anti-PTK2, 1:1000, ab40794, Abcam), AKT(Anti-AKT, 1:1000, ab18785, Abcam), p-AKT(Anti-AKT1-phospho-S473, ab81283, Abcam), mTOR(1:1000, 66888-1-Ig, Proteintech), pmTOR(Anti-mTOR-phospho S2448, ab109268, Abcam), GAPDH (1:1000, 60004-1-Ig, Proteintech), followed by reaction with HRP-conjugated secondary antibodies (1:1000, Boster). ECL Western Blotting Substrate (Beyotime) was applied for Western blot band detection. Images were visualized under Biorad Imaging Systems.

\section{Luciferase assay and dual-luciferase reporter system}

The plasmid psiCHECK2 contains the cloned CircC16orf62 WT and its mutant sequences. HCC cells $(4 \times$ $10^{4}$ cells/well) were cultivated in 6-well plates overnight and transfected with psiCHECK vector, psiCHECKCircC16orf62 WT, psiCHECK-CircC16orf62 mutant, with the plasmid for Renilla luciferase expression by lipofectamine 3000 . After $24 \mathrm{~h}$, the cells were lysed and luciferase activities were investigated by Dualluciferase reporter assay system (Promega, US).

\section{glucose consumption, lactate production, cellular ATP level}

Glucose consumption and lactate production were detected with the assay kit purchased from Beyotime (Shanghai, China). The glucose and lactate levels in the 
medium were measured using a glucose assay kit and a lactate assay kit according to the instruction manual respectively. The level of glucose and lactate were normalized to corresponding cells number. For the cellular ATP level production was analyzed by the CellTiter-Glo 2.0 Assay kit according to the manufacturer's instructions. The relative intracellular ATP level was normalized to the cell number of corresponding cell lysate. The Seahorse XF-96 Wave software was used to analyze the data. All values were normalized to cell numbers.

\section{Transwell invasion assay}

Transwell invasion assay was used for determining the invasion of different groups of HCC cells $\left(10^{5}\right.$ cells/well $)$ which were cultivated in serum-free medium in the top chamber that had been loaded with Matrigel. Seven hundred microliters of complete medium was in the bottom chambers. Twenty-four hours later, the cells on the upper surface of the top chamber were removed with a cotton swab was used for taking the cells away from the upper surface of the top chamber, then the cells on the top chamber were dyed by Harris purple crystal solution $(0.2 \%)$ (Sigma) and photoimaged.

\section{In vivo assay}

For the in vivo assay, the HCC cells of circC16orf62 knockdown, negative control were subcutaneously injected into BALB/c nude mice (4-6-weeks old, female, $n=5$ per group). The nude mice were observed every week and euthanized in 5 weeks after injection. The tumors were dissected and weighed. all conditions and procedures for the animal experiments were approved by the Animal Care Committee of The Affiliated Cancer Hospital of Guizhou Medical University.

\section{Statistical analysis}

All the data were analyzed and performed using GraphPad prism 8.0 (La Jolla, CA, USA) or SPSS Statistics software (Armonk, NY, USA). Two groups were estimated using a student's $t$-test, and three groups and more than three groups were using the non-parametric test (Mann-Whitney test) or One-way ANOVA test. The overall survival rate was calculated according to the Kaplan-Meier analysis. ${ }^{*} p \leq 0.05,{ }^{* * *} p \leq 0.01$, and ${ }^{* * * *} p \leq$ 0.001 were considered statistically significant.

\section{Results}

\section{CircC16orf62 is upregulated in HCC and associated with progression}

We downloaded the Chip data in the GEO database for screening the differently expressed circRNAs in the HCC tissues and adjacent tissue. The results indicated that the expression of circC16orf62(circRNA_0005699) was higher in tumor tissues than in adjacent normal ones which can be seen from Fig. 1A. To solidify results, 88 pairs were studied the expression level of circC16orf62 by qRT-PCR. It is indicated that circC16orf62 was overexpressed in the HCC tumor tissue than in adjacent normal tissues (Fig. 1B). The FISH assay evaluated the expression, the result indicated that the expression of circC16orf62 was higher in the $\mathrm{HCC}$ tissue than in the adjacent tissue (Fig. 1C). Then, survival analysis assessed the circC16orf62 value in diagnose and we found that circC16orf62 expression was negatively associated to prognosis (Fig. 1D). The measurement was performed on circC16orf62 in the healthy liver epithelial cell line LO2 and in $7 \mathrm{HCC}$ cell lines, including BEL7402, QGY7701, SMMC7721, HepG2, Hep3B, Huh7. This circRNA expressed much higher in human HCC cell lines than in normal liver epithelial (Fig. $1 \mathrm{E})$. Table 1 indicates the relation between the expression and clinical parameters. No obvious distinguishment in the expression of this circRNA between the two groups when taking age, gender, cirrhosis or HBV virus infection into consideration. The expression was vitally associated to Diameter of tumor $(p<0.05)$ and lymph node metastasis $(p<0.05)$. The above suggested this circRNAs was upregulated in HCC and may related to tumorigenesis and its advance.

\section{The characteristics of the circC16orf62}

Investigation on the characteristics of circC16orf62 need to be carried out before delving into the speciality of that in HCC. Figure 2A shows the genomic locus of circC16orf62. The spliced mature sequence length is $1198 \mathrm{bp}$. For that head-to-tail splicing is the result of reverse splicing of cDNA and genomic rearrangements, convergent primers and divergent primers were created for circC16orf62 to perform PCR using cDNA or genomic DNA from SMMC7721 cells as the template. Fig. 1C shows that circC16orf62 was amplified by the divergent primers only from cDNA but not from gDNA. Further, RNase $\mathrm{R}$ exonuclease and actinomycin $\mathrm{D}$ examine the authenticity of circC16orf62 in Hep3B and SMMC7721 cells. Figure 2C, D shows that circC16orf62 was resistant to RNase $\mathrm{R}$ and actinomycin $\mathrm{D}$, whereas C16orf62 line RNA was significantly inhibited after RNase $\mathrm{R}$ and actinomycin $\mathrm{D}$ treatment. The cytoplasmic nuclear isolation experiment and RNA FISH assays illustrated that most of the circC16orf62 can be found in the cytoplasm (Fig. 2E, F). Data above illustrated that the circC16orf62 samples was indeed circular and mainly located in the cytoplasm.

circC16orf62 promotes the proliferation, metastasis, and glycolysis of HCC cells

To found the biological role of circC16orf62 in HCC cells, we constructed the circC16orf62 shRNA and 


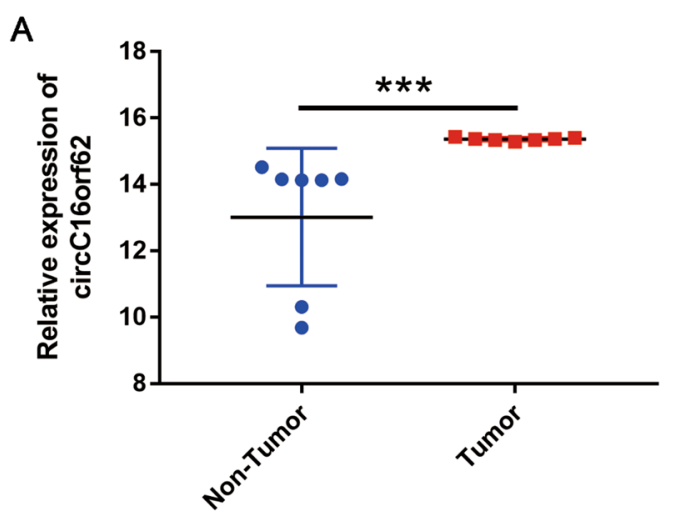

B

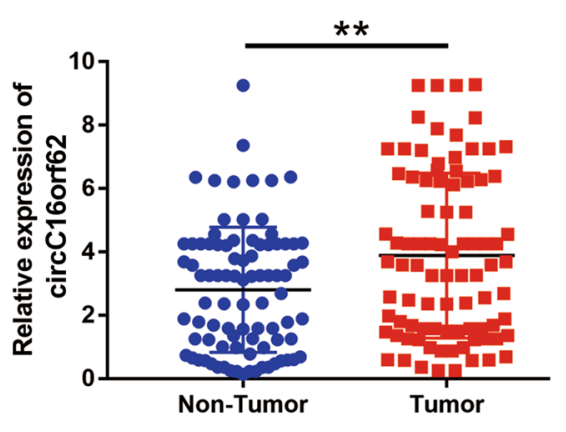

C

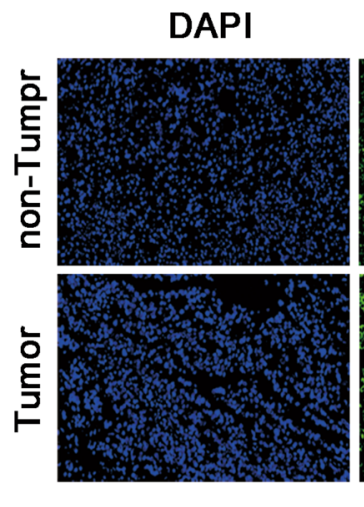

CircC16orf62

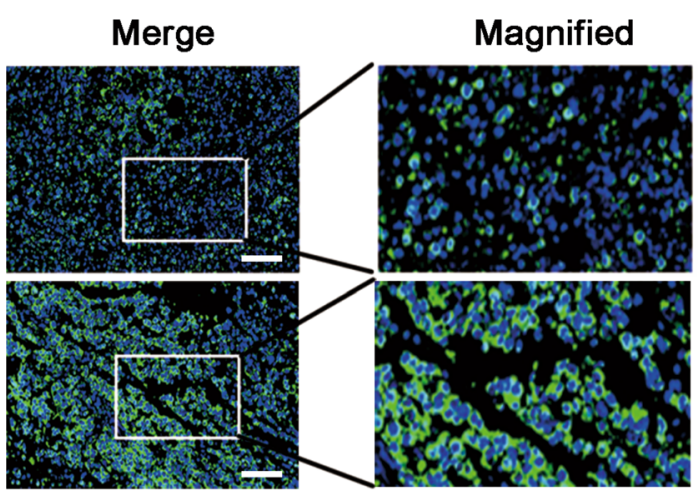

D

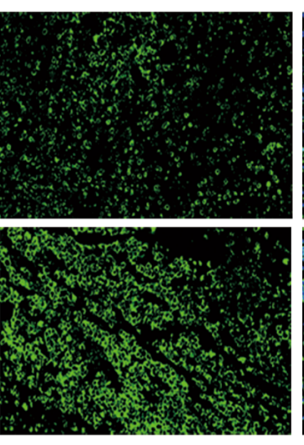

E
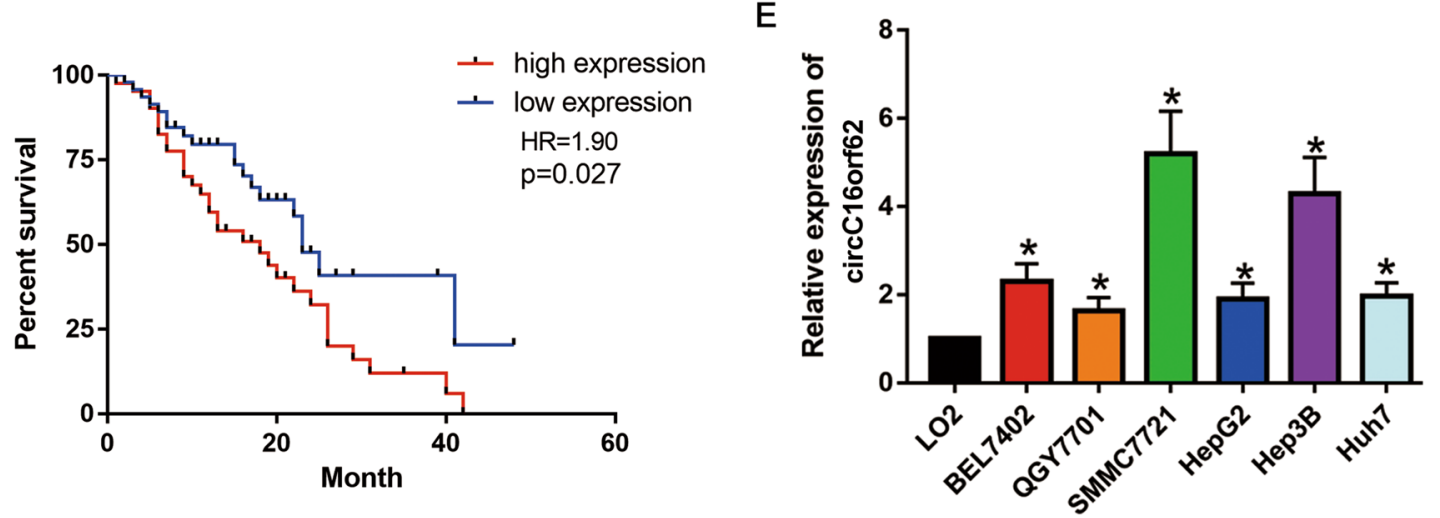

Fig. 1 CircC16orf62 is upregulated in HCC and associated with progression. A Bioinformation analysis the circC160rf62(circRNA_0005699) expression in the HCC tissues and adjacent tissue based on the GEO database (GSE97332). B q-PCR analysis the expression level of circC16orf62 in tumor tissues and adjacent normal tissues. C FISH assay was used to examine the expression of circC16orf62 in the HCC tissue, the scale bar represents $50 \mu \mathrm{m}$. D Kaplan-Meier analysis showed that the expression of circC16orf62, was predictive of overall survival in HCC. E qRT-PCR to detect the expression of circC16orf62 in six human HCC cell lines and one human non-cancerous cell line (LO-2). Data are shown as the mean \pm standard deviation of three independent experiments.* $P<0.05$ was regarded as statistically significant.

transfected the plasmid into the HCC cells Hep3B and SMMC7721. qRT-PCR verifies the transfection efficiency (Fig. 3A). Then, we used CCK-8 and plate colony assays to find the proliferation ability and evaluated the invasion and migration of the HCC cells by the transwell assays. Fig. 3B-E indicated that knockdown of circC16orf62 noticeably inhibited both the proliferation, Fig. 3F, G shows the invasion and migration ability of HCC cells.
Glucose consumption, lactate production, pyruvate production, and ATP level were examined in circC16orf62 downregulated and their control respectively. circC16orf62 can increase glucose consumption (Fig. 3H), lactate production (Fig. 3I), pyruvate production (Fig. 3J), and decrease ATP level (Fig. 3K). Then, the western blot and q-PCR indicated the aerobic glycolysis enzymes were downregulated in the circC16orf62 knockdown group. 
Table 1 Correlation between circC16orf62 expression and clinicopathological characteristics of HCC patients.

\begin{tabular}{|c|c|c|c|c|}
\hline \multirow{2}{*}{$\begin{array}{l}\text { Clinical epidemiology and } \\
\text { clinicopathologic feature }\end{array}$} & & \multicolumn{2}{|c|}{ circC16orf62 } & \multirow[t]{2}{*}{$p$ value } \\
\hline & & High & $\begin{array}{l}\text { Low } \\
\text { expression }\end{array}$ & \\
\hline All cases & & 56 & 32 & \\
\hline \multicolumn{5}{|l|}{ Age } \\
\hline & $\leq 50$ & 21 & 17 & 0.183 \\
\hline & $>50$ & 35 & 15 & \\
\hline \multicolumn{5}{|l|}{ Gender } \\
\hline & Male & 31 & 12 & 0.125 \\
\hline & Female & 25 & 20 & \\
\hline \multicolumn{5}{|l|}{ Diameter of tumor } \\
\hline & $\leq 4$ & 25 & 23 & $0.016^{*}$ \\
\hline & $>4$ & 31 & 9 & \\
\hline \multicolumn{5}{|l|}{ HBV virus infection } \\
\hline & Negative & 24 & 18 & 0.271 \\
\hline & Positive & 32 & 14 & \\
\hline \multicolumn{5}{|l|}{ Cirrhosis } \\
\hline & Negative & 16 & 11 & 0.634 \\
\hline & Positive & 40 & 21 & \\
\hline \multicolumn{5}{|l|}{ Distant metastasis } \\
\hline & Negative & 45 & 27 & 0.777 \\
\hline & Positive & 11 & 5 & \\
\hline \multicolumn{5}{|l|}{ Lymph node metastasis } \\
\hline & Negative & 39 & 29 & $0.033^{*}$ \\
\hline & Positive & 17 & 3 & \\
\hline \multicolumn{5}{|l|}{ TNM stage } \\
\hline & $|/| \mid$ & 42 & 27 & 0.421 \\
\hline & III/IV & 14 & 5 & \\
\hline
\end{tabular}

${ }^{*} p$ values that are statistically significant are shown in bold.

These results implied that circC16orf62 could promote proliferation, metastasis, and aerobic glycolysis in HCC cells.

\section{MiR-138-5p was the potential target of circC16orf62 in HCC cells}

To figure out the role of circC16orf62, we made the prediction on the potential targeting miRNAs in CSCD (http://gb.whu.edu.cn/CSCD/) database and target genes (starbase) by bioinformatics. Furthermore, KEGG pathway analysis the downstream signal pathway eluted the
PI3K/AKT was the significantly downstream pathway of circC16orf62 (Fig. 4A). Then, the western-blots result demonstrated that circ C16orf62 knockdown could make inhibition on the p-AKT and p-mTOR expression (Fig. $4 \mathrm{~B})$. Based on the PI3K/AKT pathway, we constructed the ceRNA regulation network to select the targeted miRNAs (Fig. 4C). Among the 15 miRNAs, miRNA-296-5p, miRNA-138-5p, and miRNA-4282 were significantly downregulated in the HCC tissues (Fig. 4D). Further, the luciferase activity assay indicated that the miRNA-138 could inhibit the luciferase activity of circC16orf62 $3^{\prime}$ UTR (Fig. 4E). The q-RT-PCR assay detected the expression of circC16orf62 and miR-138-5p, Person analysis the expression level illustrated that the circRNA and miRNA mentioned were negatively correlated in the HCC tissues (Fig. 4F). To verified the location of the two circRNAs, the FISH assay pointed out that two types of circRNA were mainly co-located in the cytoplasm (Fig. 4G). Bioinformation analysis the binding site of the two, the corresponding mutation site was shown in Fig. $4 \mathrm{H}$. The luciferase activity assay indicated that miR-138-5p could combine with circC16orf62 (Fig. 4I). Then, the RIP assay indicated that Ago2 could combine with the two, the results indicated that they were acted on the Ago2 (Fig. $4 \mathrm{~J})$. Together, the results above show that circC16orf62 perhaps sponge miR-138-5p in HCC cells.

\section{MiRNA-138-5p targets the PTK2 expression involved in AKT/mTOR signal pathway}

The target genes with the potentiality of miRNA-138-5p were picked out by the prediction software(PicTar, miRTargetbase, Targetscan, miRDB). Based on the bioinformatic analysis, PTK2 was found as the putative target gene (Fig. 5A and Supplementary Material 1). Based on the TCGA database, the results indicated that PTK2 was upregulated in the HCC (Fig. 5B). Figure 5C shows the person analysis demonstrated that miRNA-138-5p and PTK2 were negatively correlated in HCC tissues. Furthermore, the q-RT-PCR and western-blot assay indicated that miRNA could inhibit that PTK2 expression in HCC cells (Fig. 5D, E). Then, the bioinformation analysis found the binding site of miRNA-138-5p and PTK2, the luciferase wide type and mutation type plasmid was constructed according to the binding site (Fig. 5F). The luciferase activity assay was used to verify the combination of miRNA-138-5p and PTK2, the result indicated that miRNA-138-5p could bind with the PTK2 $3^{\prime}$ UTR (Fig. 5G, H). Then, the western blots indicated that circC16orf62 knockdown inhibited AKT/mTOR signaling pathway, and the effect could partly be inhibited by miR138 inhibitor or PTK2 (Fig. 5I). Data above indicated that PTK2 was the potential function target of miRNA-138-5p in the progress of $\mathrm{HCC}$. 
A
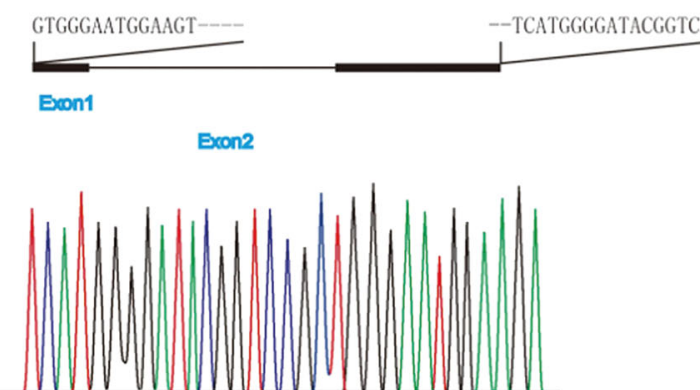

TCATGGGGATACGGTCCGGTGGGAATGGAAGT--

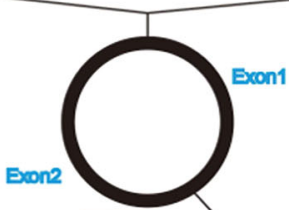

D

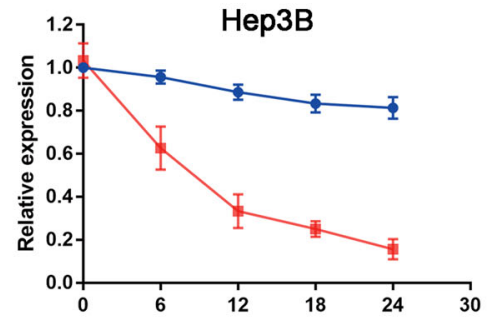

E
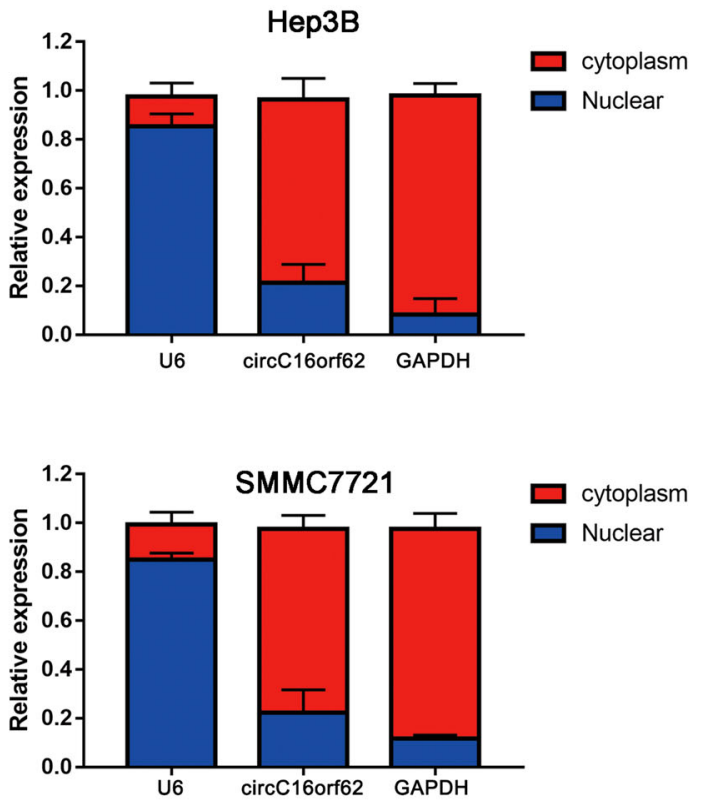

B

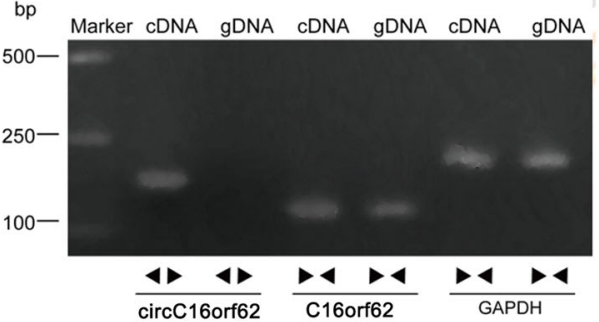

C
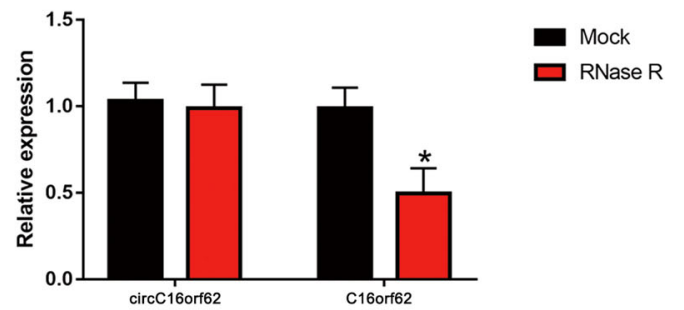

SMMC7721

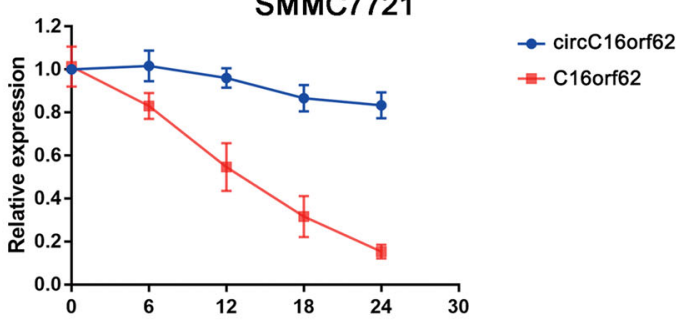

F
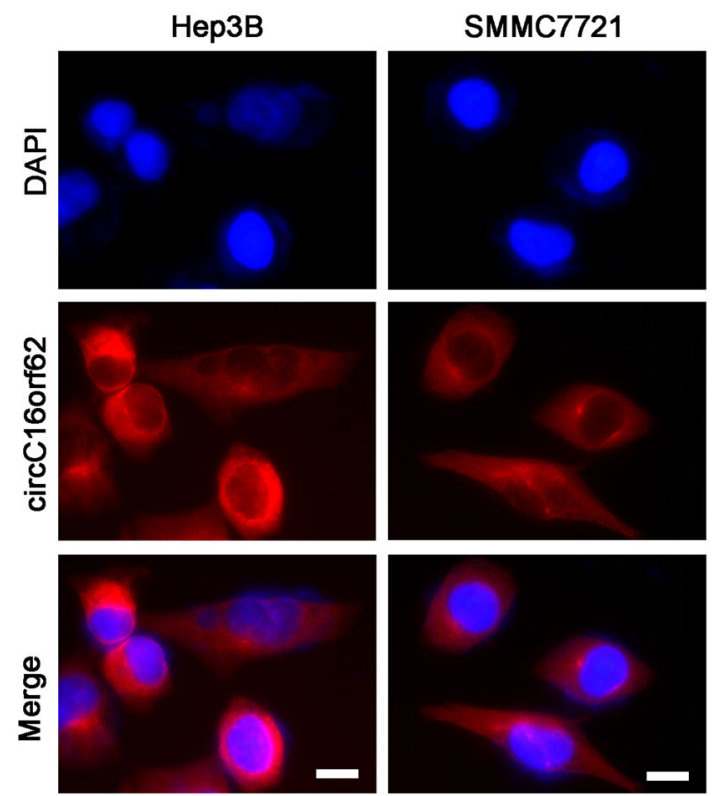

Fig. 2 The characteristics of the circC16orf62. A circUBA1 originates from back-spliced exons 1 to 2 of C16orf62. The back-splice junction of circC16orf62 was identified by Sanger sequencing. B PCR analysis for circC16orf62 and linear C16orf62 in cDNA and gDNA. C The stability of circC16orf62 was evaluated by RNase R digestion assay. D PCR analysis for circC16orf62 and linear C16orf62 in CDNA and gDNA by RNase R digestion assay. E Nucleoplasmic separation analysis the location of circC16orf62. F FISH assays showed that circC16orf62 was distributed principally in the cytoplasm, the scale bar represents $10 \mu \mathrm{m}$. Data are shown as the mean \pm standard deviation of three independent experiments. ${ }^{*} P<0.05 \mathrm{was}$ regarded as statistically significant. 
A

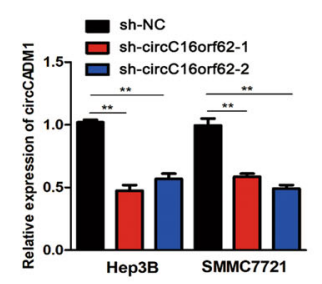

D

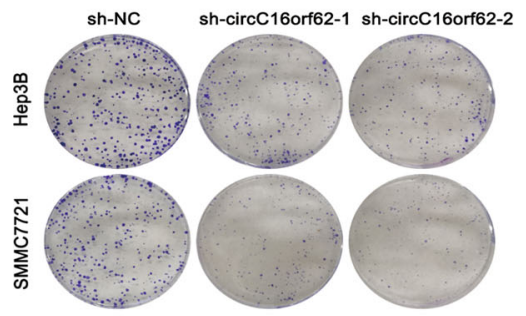

$\mathbf{F}$
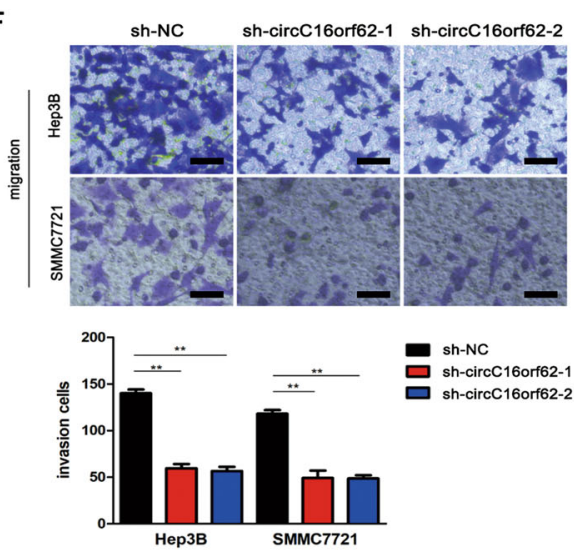

$\mathrm{H}$

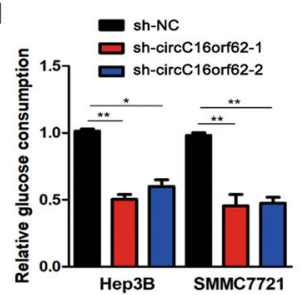

L

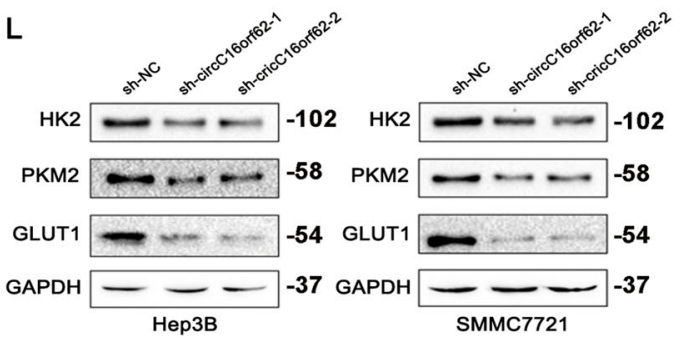

C

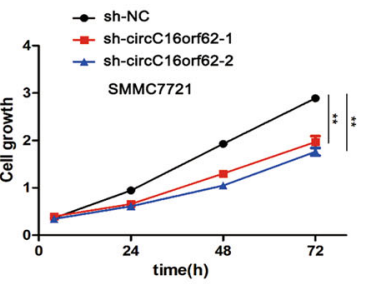

E

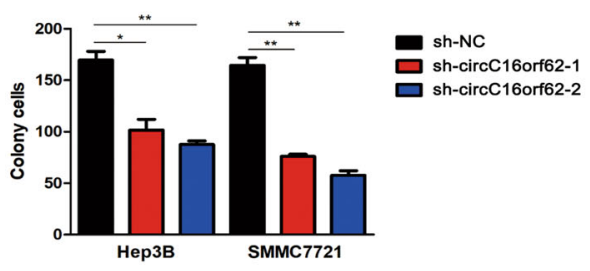

G
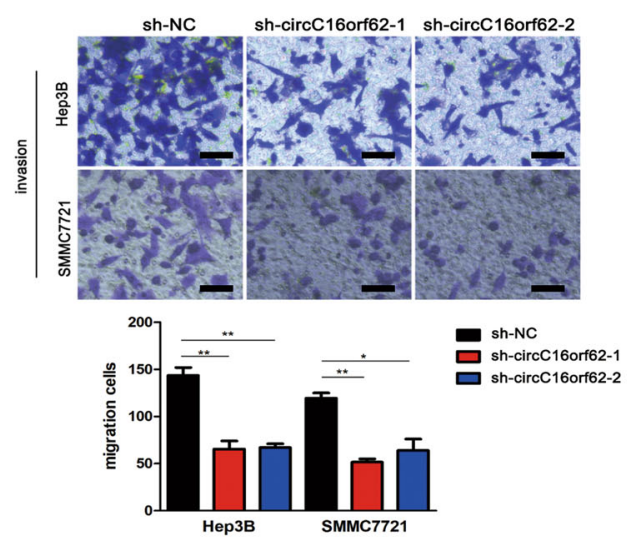

J

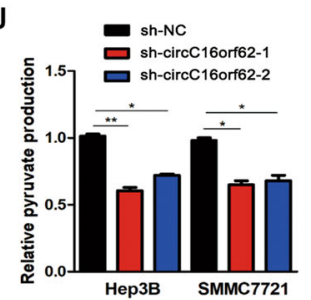

K

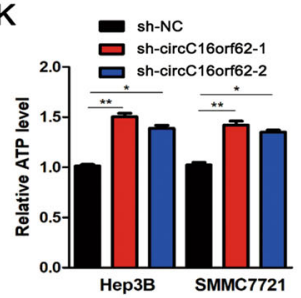

M

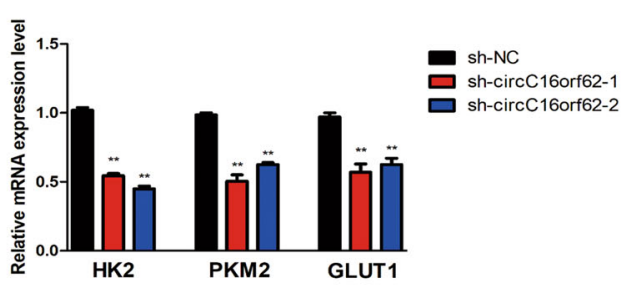

Fig. 3 circC16orf62 promotes the proliferation, metastasis, and glycolysis of HCC cells. A The effects of knockdown or overexpression of circC16orf62 in Hep3B or SMMC7721 cells were measured using qRT-PCR. B-E CCK-8 and colony formation assays of PC cells with circC16orf62 knockdown. F, G Transwell assays showed that invasion and migration were significantly inhibited after circC16orf62 knockdown, the scale bar represents $50 \mu \mathrm{m}$. H-K Glucose consumption $(\mathbf{H})$, lactate production (I), pyruvate production (J), ATP production (K) were measured in circC16orf62 knockdown HCC cells. L, M RT-qPCR and western blot analysis several metabolic enzymes (GLUT1, PKM2, HK2) expression level were in circC16orf62 knockdown HCC cells. Data are shown as the mean \pm standard deviation of three independent experiments. ${ }^{*} P<0.05$ was regarded as statistically significant. 


\section{A}

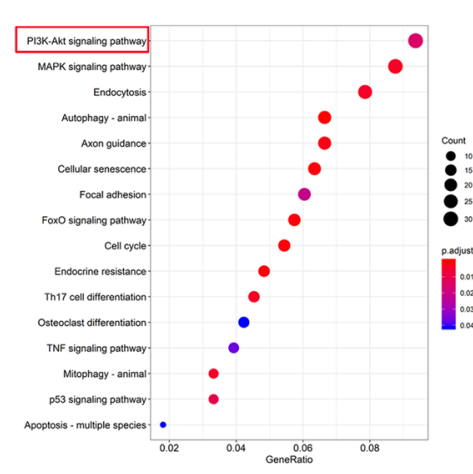

B

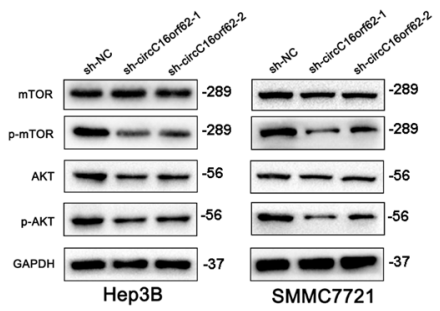

C

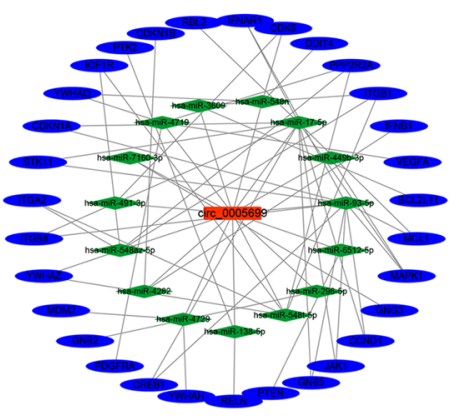

PI3KJAKT ceRAN network
D

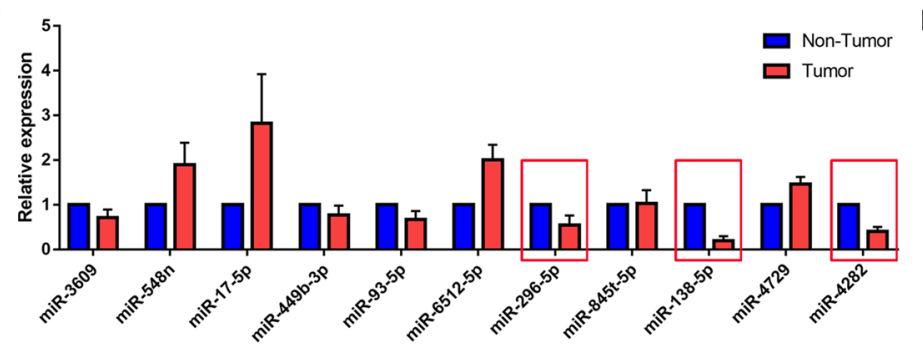

E

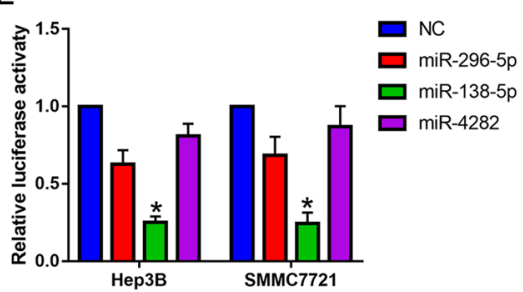

F

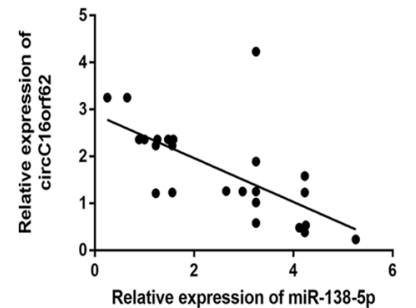

$p<0.05$
$R=0.43$

G DAPI

circC16orf62
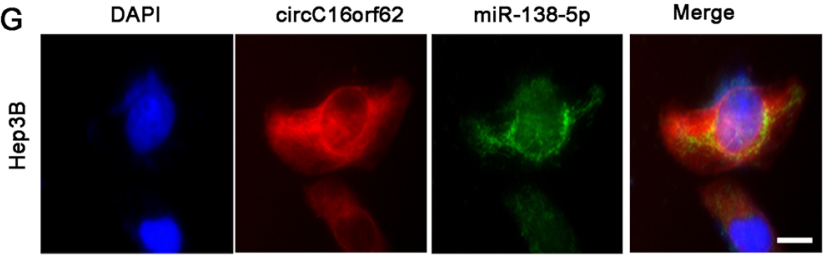

$\mathrm{H}$
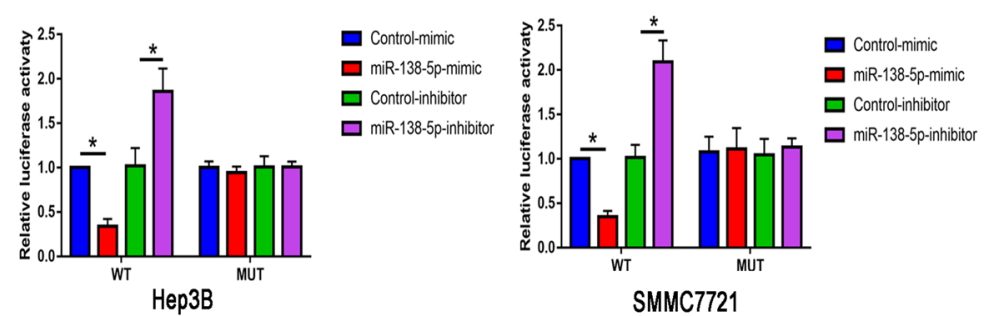

J
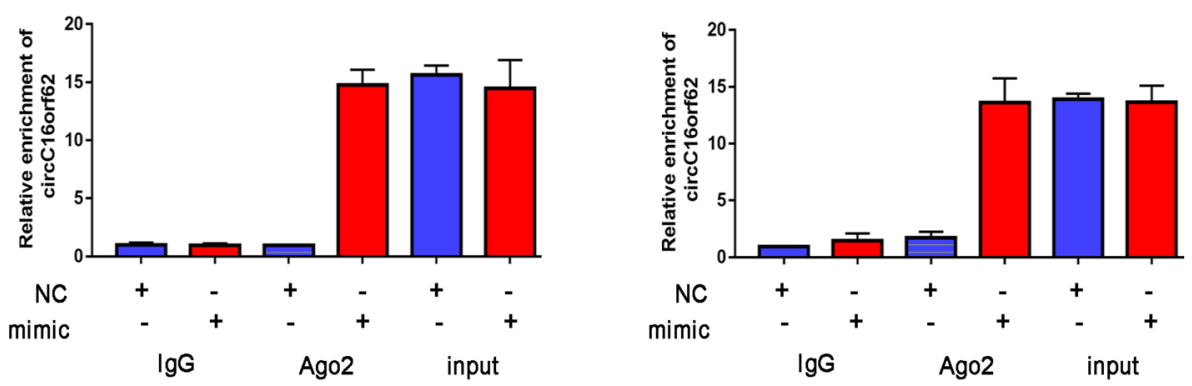

Fig. 4 Bioinformatic analysis and validation of potentially targeted miRNAs of circC16orf62 in HCC cells. A KEGG pathway analysis the downstream signal pathway eluted the PI3K/AKT was the significantly downstream pathway of circC16orf62. B Western-blots analysis the expression of p-AKT and p-mTOR. C ceRNA network analysis the target miRNAs of circC16orf62. D RT-qPCR and western blot analysis the target miRNAs in the HCC tumor and adjacent normal tissue. E Luciferase analysis the interaction of circC16orf62 and miRNA-138. F RT-qPCR analysis the expression correlation of circC16orf62 and miRNA-138 in the HCC tumor and adjacent normal tissue. G FISH assay analysis the circC16orf62 and miRNA-138 co-location in the Hep3B cell, the scale bar represents $10 \mu \mathrm{m}$. $\mathbf{H}$ TargetScan showed that circC16orf62 could bind to the miR-138-5p. I The direct binding between circC16orf62 and miRNA-138 was analyzed by dual-luciferase reporter assay. J A RIP assay was performed using an AGO2 antibody, and lgG served as a negative control. Data are shown as the mean \pm standard deviation of three independent experiments. ${ }^{*} P<0.05$ was regarded as statistically significant. 


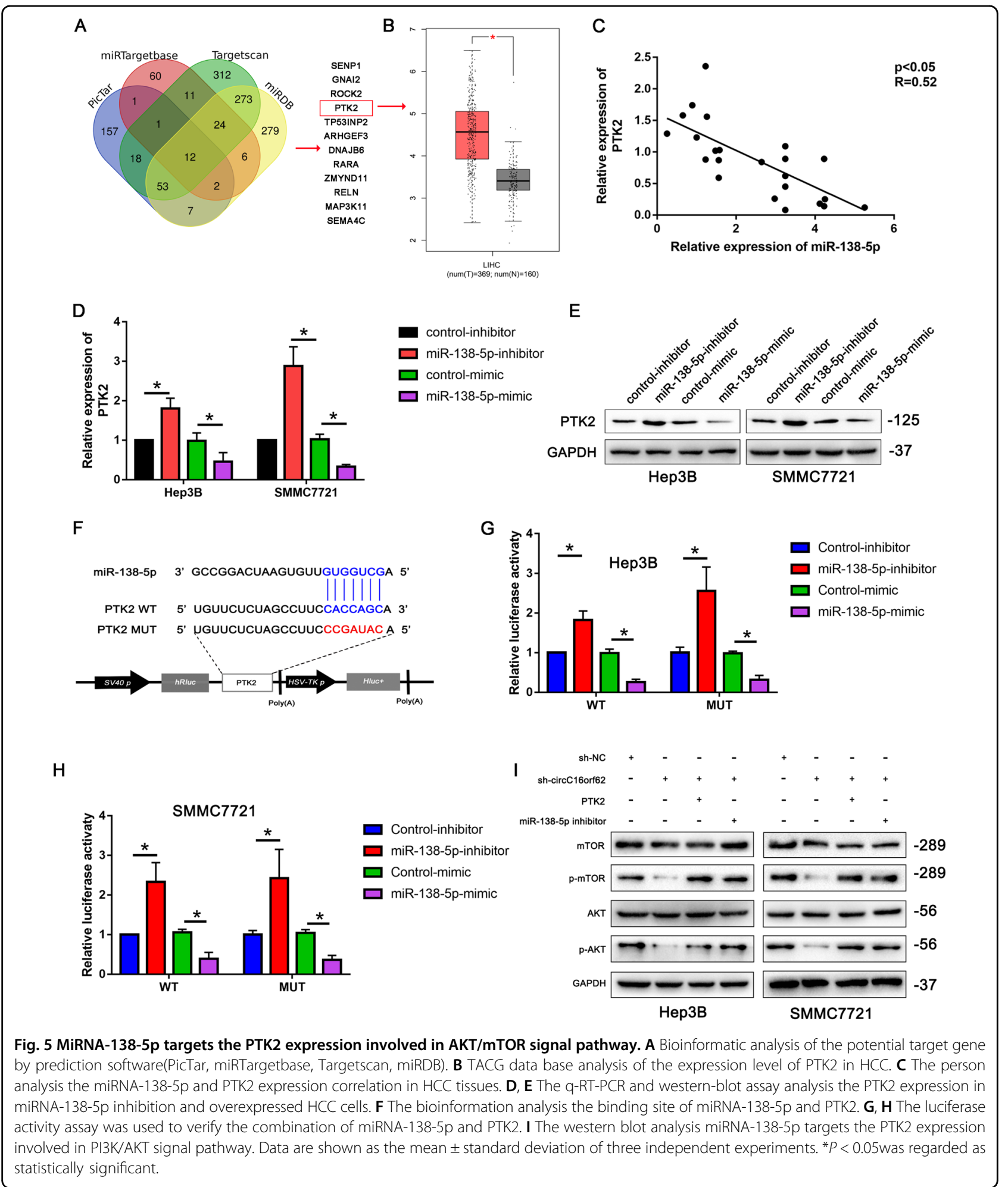

circC16orf62 knockdown repressed HCC progression via controlling MiR-138-5p/PTK2

HCC cells were transfected with sh-circC16orf62, and co-transfected with miR-138-inhibitor or PTK2 overexpressed plasmid. The CCK8 and plate colony assay indicated that circC16orf62 could promote the growth ability of HCC, and miR-138 inhibitor and PTK2 overexpress plasmid could partly reverse the effect of 
circC16orf62 (Fig. 6A-C). And the transwell assay indicated that the migration and invasion of $\mathrm{HCC}$ cells were upregulated in the cell that transfected with miR-138inhibitor or PTK2 overexpressed plasmid (Fig. 6D, E). Then, MiR-138-5p/PTK2 could increase glucose consumption (Fig. 6F), lactate production (Fig. 6G), pyruvate production (Fig. $6 \mathrm{H}$ ), and decrease ATP level in the HCC cells transfected with circC16orf62 shRNA (Fig. 6I). These data eluted that circC16orf62 knockdown repressed HCC progression via regulating MiR-138-5p/PTK2.

\section{Knockdown of circC16orf62 shows growth inhibition of $\mathrm{HCC}$ cell in vivo}

To figure out the role of circC16orf62 in HCC growth in vivo, we injected Hep3B cells transfected with $\mathrm{NC}$ or circC16orf62 shRNAs(sh-circC16orf62-1, sh-circC16orf622) to the mice. Knockdown of sh-circC16orf62 reduced the weight of the tumor after 5-week subcutaneous injection (Fig. 7A-C). KI-67 and PCNA staining assured that lower growth ability presents in the mice which were with SMMC7721 cells transfected with sh-circC16orf62 shRNA (Fig. 7D). IHC detects positive expression of PTK2, p-AKT, p-MTOR, PKM2, GLUT1, HK2. And expression levels of the target gene PTK2, the AKT signal indicator p-AKT, and p-MTOR, the glycolysis target PKM2, GLUT1 and HK2 were downregulated through the transfection of circC16orf62 shRNA (Fig. 7E). All in all, circC16orf62 serves as an activator of osteosarcoma progression in vivo.

\section{Discussion}

HCC cancer is the commonest malignant tumor that patients has increased year by year ${ }^{18}$. Endoscopic minimally invasive surgery and surgical eradication can be used to deal with early $\mathrm{HCC}^{19,20}$. For no significant symptoms of most cases, almost $60 \%$ of HCC has progressed when diagnose with lymph node and distant metastases (into the middle and late stages) ${ }^{21}$. Early detection and treatment lead to a better prognosis. For that, finding an effective novel biomarker and exploring HCC pathogenesis-related signaling pathways is vital ${ }^{22,23}$.

Based on the GEO database, we analysis the different circRNAs in seven pairs HCC tissue and corresponding adjacent normal tissues. We figure out the differently expressed circRNAs in HCC tissues and corresponding adjacent normal tissues. The circRNAs could be biomarkers with potentiality and therapeutic targets for the diagnosis HCC. Based on our data, selected circRNAs exhibit vital distinguishment in expression and they were measured in additional samples. The expression level of it was obviously related to clinical stage and malignancy. survival analysis pointed out that circC16orf62 in HCC may be a promising prognostic biomarker. Various functional experiments suggested that knockdown of the
circRNA mentioned vitally inhibits the proliferation and invasion of the cancer cells, as the mentioned one functions likely an oncogene. Especially, the circC16orf62 expression was obviously enhanced in the HCC bigger tumor size and distal metastasis group. When taking the effect on the invasion of HCC cells in the human body into consideration, circC16orf62 functions vital in the malignant progression.

The miRNA sponge mechanism is a foundation for detecting the bio-functions of circRNAs ${ }^{24}$. Accumulated literature discovered that circRNA could function as a miRNA sponge. The RIP assay confirmed that circC16orf62 adsorbs miRNA. So we used target prediction software to predict circRNA-miRNA-mRNA interactions through and built associated networks. Dual-luciferase reporter assays found that circC16orf62 could directly interact with miR-138-5p (predicted target). The rescue experiments indicated that decreased HCC cell proliferation and invasion resulted from circC16orf62 knockdown offset for miR-138-5p inhibition. A report indicated that this miRNA functions like a tumor suppressor in many cancers ${ }^{25,26}$, and the interaction of the two attenuates the tumor suppressor efficiency of miR138-5p. circC16orf62 is like an oncogene, sponging miR138-5p in HCC.

PI3K/AKT signaling is widely activated in human cancers, and investigation on it has been well performed ${ }^{27}$. In addition, some cancers contain overexpression of PTK2 which reported function as oncogene ${ }^{28}$. PTK2 mRNA and protein expression in HCC tissues were higher in our data than those in adjacent normal tissues which vital positive relationship with the expression of circC16orf62. The function assay indicated that PTK2 acted as an activator to assist the promoting effect exerted by circC16orf62 in HCC cell proliferation and invasion. Meanwhile, PTK2 was known as a protein kinase to activte AKT/mTOR signaling pathway, Can play a regulatory role in the occurrence and development of tumors. Function recovery experiment found that the miR-138-5p inhibitor partly restrained the effect of circC16orf62 inhibition on cell proliferation. Abnormal activation of AKT-mTOR signaling pathway functions vital in the malignant progression of HCC. The results indicated that downregulation of circC16orf62 noticeably inhibit the expression level of PTK2 which further mediating AKT/ mTOR signaling activating in HCC. So the circC16orf62 /miR-138-5p/PTK2/AKT signaling pathway functions vital in HCC. In conclusion, circC16orf62, a biomarker with much potential, is upregulated human HCC issues. The effect of the circC16orf62/miR-138-5p/PTK2/AKT regulation network on the progress and deterioration of HCC. We believe that circC16orf62 is a therapeutic target with potential for HCC. 


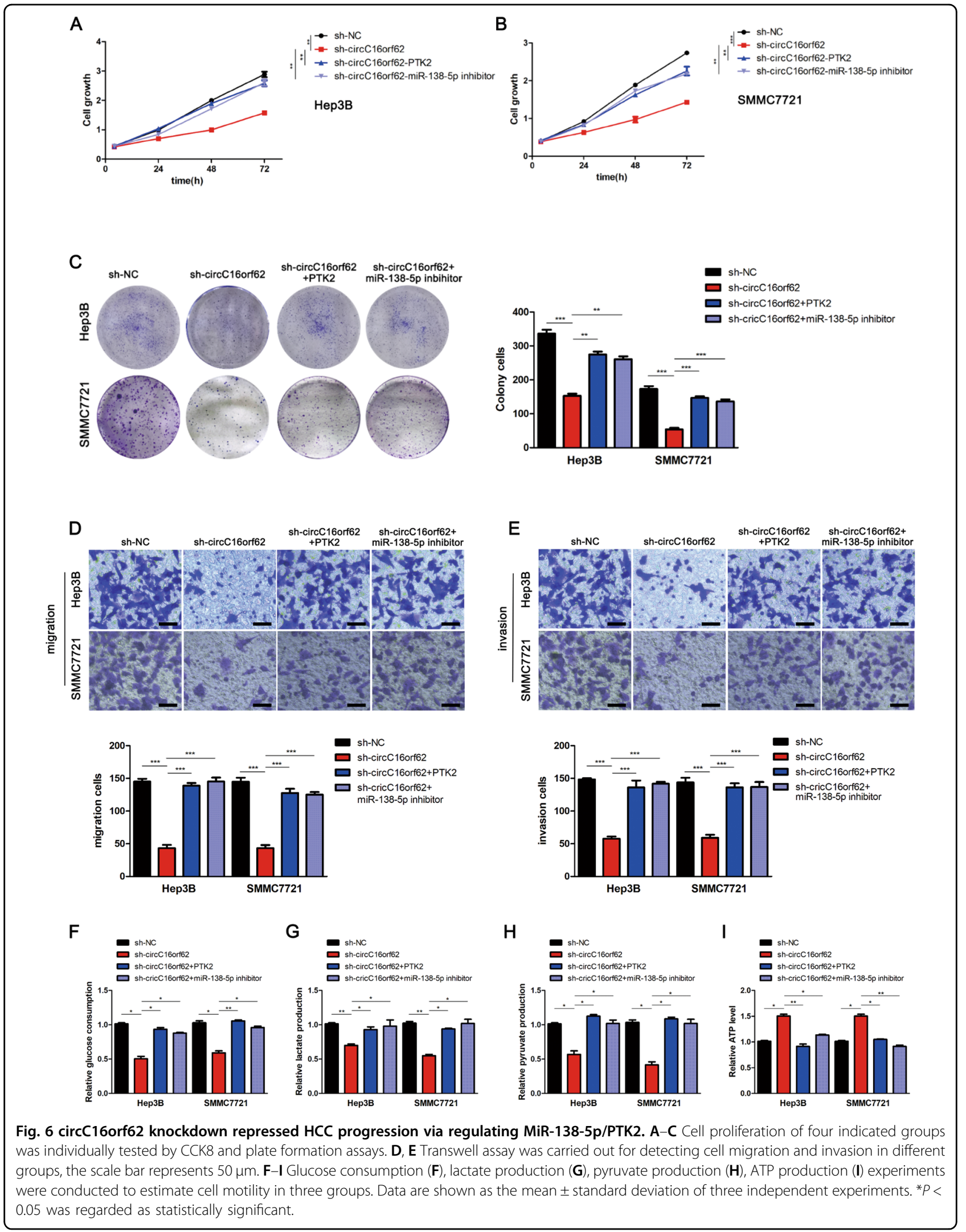



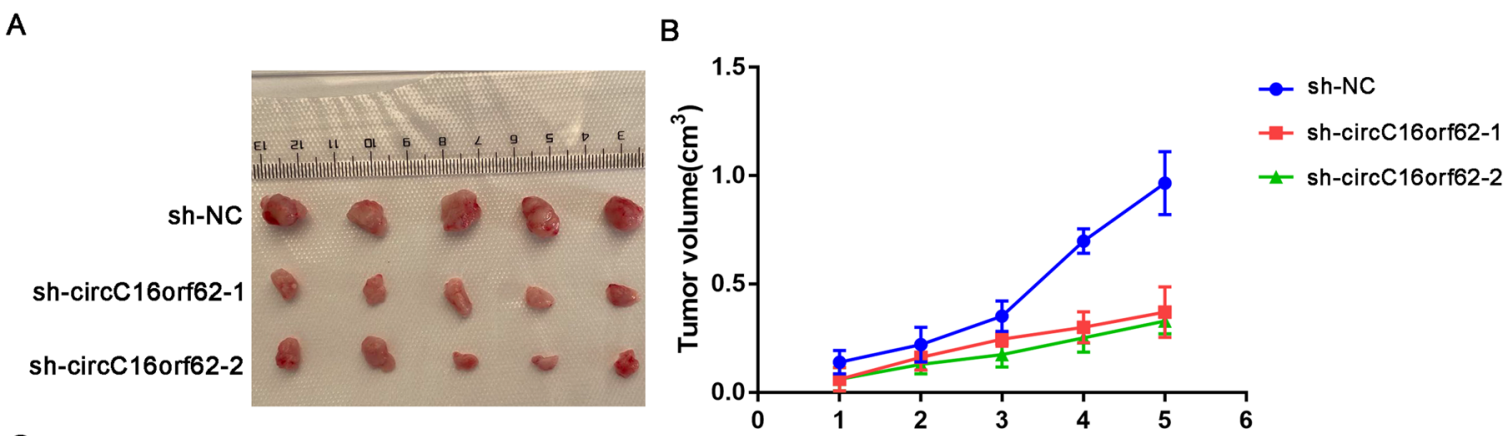

C

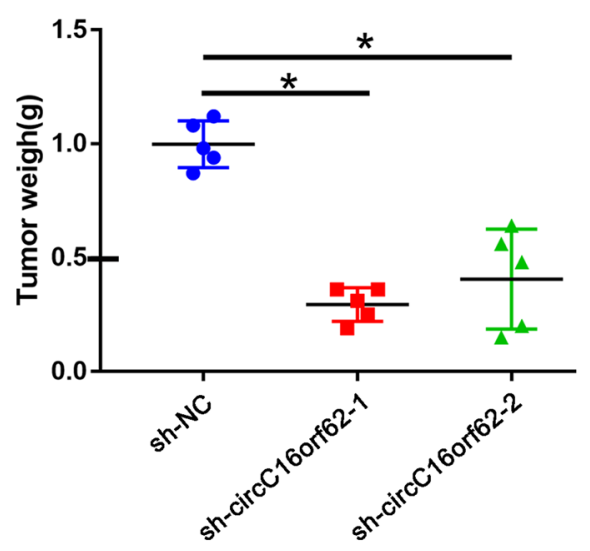

D

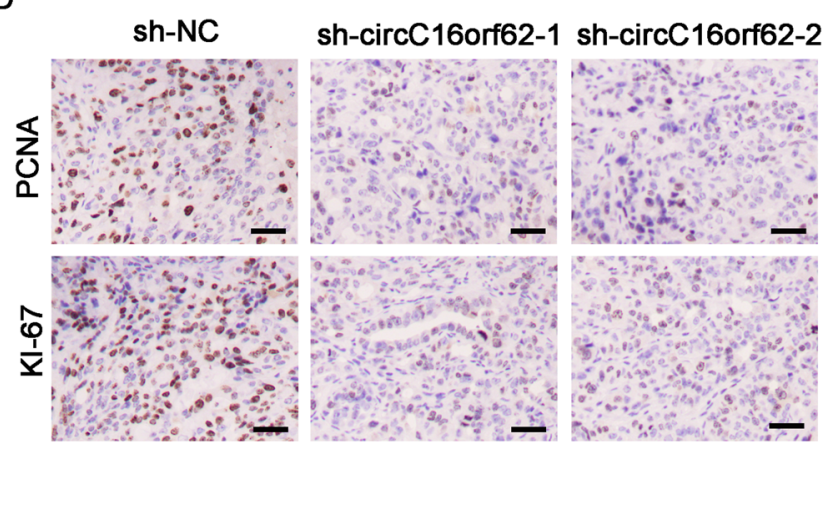

E
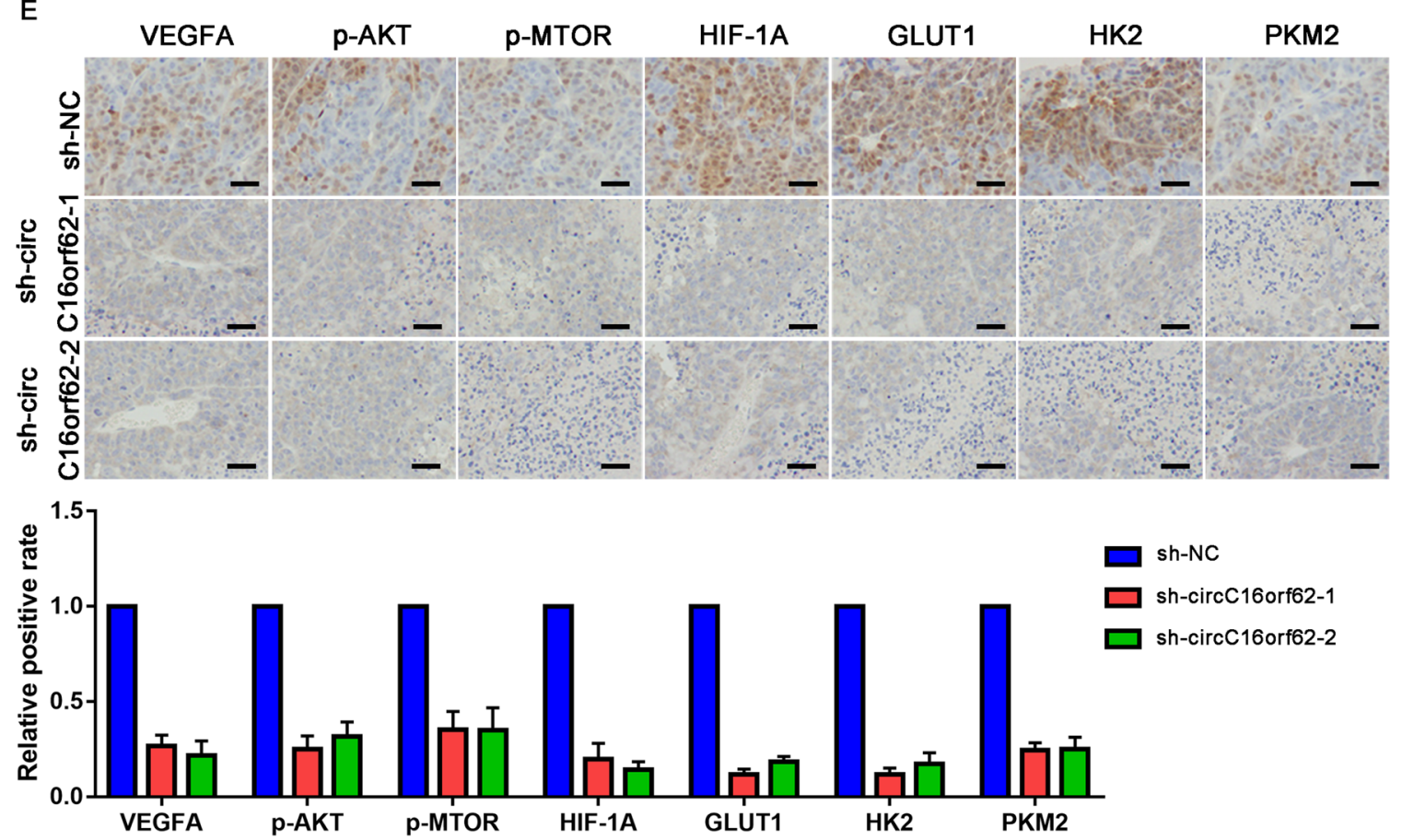

Fig. 7 Knockdown of circC16orf62 inhibited in vivo growth of HCC cell. A The BALB/c nude mice were sacrificed for the xenografts, and the size was measured by the beside ruler. B The tumor growth curve of xenografts was plotted in shNC and shcircC16orf62 group ( $n=5$ each group) by measuring the tumor size (width ${ }^{2} \times$ length $\times 0.5$ ) with vernier caliper. $\mathbf{C}$ The anatomized subcutaneous tumor xenografts were weighed and analyzed with student's $t$ test. $\mathbf{D} \mathbb{H C}$ analysis the proliferation markers (PCNA and KI-67) in different group xenografts, the scale bar represents $100 \mu \mathrm{m}$. $\mathbf{E} 1 \mathrm{HC}$ analysis the AKT/mTOR signaling pathway markers in different group xenografts, the scale bar represents $100 \mu m$. Data are shown as the mean \pm standard deviation of three independent experiments. ${ }^{*} P<0.05$ was regarded as statistically significant. 


\section{Acknowledgements}

We acknowledge the generous support of the Department of Interventional Radiology, the Affiliated Hospital of Guizhou Medical University, Guiyang, Guizhou, China.

\section{Author details}

'Department of Interventional Radiology, The Affiliated Cancer Hospital of Guizhou Medical University, Guiyang 550000, P.R. China. ${ }^{2}$ Department of Interventional Radiology, the Affiliated Hospital of Guizhou Medical University, Guiyang 550004, P.R. China. ${ }^{3}$ Department of Cancer Research Laboratory, The Affiliated Cancer Hospital of Guizhou Medical University, Guiyang 550000, P.R. China. ${ }^{4}$ Guizhou Provincial Key Laboratory of Pharmaceutics, Guizhou Medical University, Guiyang 550004, P.R. China. ${ }^{5}$ School of Basic Medical Sciences, Guizhou Medical University, Guiyang 550004, P.R. China. ${ }^{6}$ Department of Radiology, the Affiliated Hospital of Guizhou Medical University, Guiyang 550004, P.R. China. 'Department of Interventional Radiology, First Affiliated Hospital of Guizhou University of Traditional Chinese Medicine, Guiyang 550000, P.R. China

\section{Author contributions}

S.Z., R.L., S.Z. contributed to the experiment design, and data analysis. Y.L., Z.-J W., Y.-H.W., T.L., B.-J.X., X.-Y.Z., Y.H., X.Z. contributed to the experiment implementation, S.Z. contributed to manuscript draft and data analysis. H.-Y.J., Zhi-M.C., S.Z. read and approved the final manuscript. We acknowledge the generous support of the Department of Interventional Radiology, the Affiliated Hospital of Guizhou Medical University, Guiyang, Guizhou, China.

\section{Funding}

The present study was supported by funds from the Natural Science Foundation of China (no. 81860320; no. 81960328; no. 81760325; no. 81860718), the Guizhou Province Science and Technology Projects (Guizhou foundation [2018] 1129; Guizhou foundation [2017] 1150, and the Funding for innovation and Entrepreneurship of high-level overseas students in Guizhou Province [2019 01] and the China Postdoctoral Science Foundation (no. 2020M683660XB) and Guiyang Science and technology projects ([2019]9-2-47).

\section{Data availability}

All data generated and analysed during this study are included in this published article are available on request.

\section{Ethics statement}

This study was approved by the Ethics Committee of The Affiliated Cancer Hospital of Guizhou Medical University, and informed consent was obtained from all participants included in the study, in agreement with institutional guidelines.

\section{Conflict of interest}

The authors declare no competing interests.

\section{Publisher's note}

Springer Nature remains neutral with regard to jurisdictional claims in published maps and institutional affiliations.

Supplementary information The online version contains supplementary material available at https://doi.org/10.1038/s41419-021-03866-7.

Received: 1 February 2021 Revised: 14 May 2021 Accepted: 17 May 2021 Published online: 09 June 2021

\section{References}

1. Chen, W. et al. Cancer statistics in China, 2015. CA Cancer J. Clin. 66, 115 (2016).

2. Chen, L. L. The biogenesis and emerging roles of circular RNAs. Nat. Rev. Mol. Cell Biol. 17, 205 (2016).
3. Qu, S. et al. The emerging landscape of circular RNA in life processes. RNA Biol. 14, 992 (2017).

4. Xu, T., Wu, J., Han, P., Zhao, Z. \& Song, X. Circular RNA expression profiles and features in human tissues: a study using RNA-seq data. BMC Genomics 18, 680 (2017).

5. Xiong, D. D. et al. High throughput circRNA sequencing analysis reveals novel insights into the mechanism of nitidine chloride against hepatocellular carcinoma. Cell Death Dis. 10, 658 (2019).

6. Wei, G., Zhu, J., Hu, H. B. \& Liu, J. Q. Circular RNAs: promising biomarkers for cancer diagnosis and prognosis. Gene 771, 145365 (2021).

7. Wang, S. et al. Circular RNAs in body fluids as cancer biomarkers: the new frontier of liquid biopsies. Mol. Cancer 20, 13 (2021).

8. Greene, J. et al. Circular RNAs: biogenesis, function and role in human diseases. Front. Mol. Biosci. 4, 38 (2017).

9. Zhang, $\mathrm{H}$. et al. The biogenesis and biological functions of circular RNAs and their molecular diagnostic values in cancers. J. Clin. Lab Anal. 34, e23049 (2020).

10. Hong, $\mathrm{H}$. et al. The novel circCLK3/miR-320a/FoxM1 axis promotes cervical cancer progression. Cell Death Dis. 10, 950 (2019).

11. Ou, R. et al. circAMOTL1 motivates AMOTL1 expression to facilitate cervical cancer growth. Mol. Ther. Nucleic Acids 19, 50 (2020).

12. He, Q. et al. circRNA circFUT8 upregulates Krupple-like factor 10 to inhibit the metastasis of bladder cancer via sponging miR-570-3p. Mol. Ther. Oncolytics 16, 172 (2020).

13. Ou, R. et al. circRNA-AKT1 sequesters miR-942-5p to upregulate AKT1 and promote cervical cancer progression. Mol. Ther. Nucleic Acids 20, 308 (2020).

14. Pu, Z., Xu, M., Yuan, X., Xie, H. \& Zhao, J. Circular RNA circCUL3 accelerates the Warburg effect progression of gastric cancer through regulating the STAT3/ HK2 axis. Mol. Ther. Nucleic Acids 22, 310 (2020).

15. Qiu, L. P. et al. The emerging role of circular RNAs in hepatocellular carcinoma. J. Cancer 9, 1548 (2018).

16. Guria, A., Sharma, P., Natesan, S. \& Pandi, G. Circular RNAs-The Road Less Traveled. Front. Mol. Biosci. 6, 146 (2019).

17. Sun, J. Y. et al. Diagnostic and prognostic value of circular RNAs in hepatocellular carcinoma. J. Cell Mol. Med. 24, 5438 (2020).

18. Faivre, S., Rimassa, L. \& Finn, R. S. Molecular therapies for HCC: looking outside the box. J. Hepatol 72, 342 (2020).

19. Han, T. S., Ban, H. S., Hur, K. \& Cho, H. S. The epigenetic regulation of HCC metastasis. Int. J. Mol. Sci. 19, 3978 (2018).

20. Kim, D. W., Talati, C. \& Kim, R. Hepatocellular carcinoma (HCC): beyond sorafenib-chemotherapy. J. Gastrointest. Oncol. 8, 256 (2017).

21. Viveiros, P., Riaz, A., Lewandowski, R. J. \& Mahalingam, D. Current state of liverdirected therapies and combinatory approaches with systemic therapy in hepatocellular carcinoma (HCC). Cancers 11, 1085 (2019).

22. Krishnan, M. S. et al. Genomic analysis of vascular invasion in hepatocellular carcinoma (HCC) reveals molecular drivers and predictive biomarkers. Hepatology 10, 1002 (2020).

23. Gnutzmann, D. et al. Transvascular therapy of hepatocellular carcinoma (HCC), status and developments. Minim. Invasive Ther. Allied Technol. 27, 69 (2018).

24. Chen, Y. J. et al. Genome-wide, integrative analysis of circular RNA dysregulation and the corresponding circular RNA-microRNA-mRNA regulatory axes in autism. Genome Res. 30, 375 (2020).

25. Qiu, H., Chen, F. \& Chen, M. MicroRNA-138 negatively regulates the hypoxiainducible factor 1alpha to suppress melanoma growth and metastasis. Biol. Open 8, 8 (2019).

26. Zhang, J. et al. MicroRNA-138 modulates metastasis and EMT in breast cancer cells by targeting vimentin. Biomed. Pharmacother. 77, 135 (2016).

27. $\mathrm{Wu}, \mathrm{Y}$. et al. PI3K/AKT/mTOR pathway-related long non-coding RNAs: roles and mechanisms in hepatocellular carcinoma. Pharmacol. Res. 160, 105195 (2020).

28. Fan, Z. et al. PTK2 promotes cancer stem cell traits in hepatocellular carcinoma by activating Wnt/beta-catenin signaling. Cancer Lett. 450, 132 (2019). 